\title{
Políticas públicas de inclusión digital: El caso de América Latina y Cuba
}

\author{
Edgar Bisset Alvarez \\ Angela Maria Grossi de Carvalho \\ Silvana Aparecida Borsetti Gregorio Vidotti \\ Universidade Estadual Paulista - UNESP, Brasil
}

\section{REVIEW}

\section{Resumen}

Objetivo. Abordar el desarrollo de políticas públicas vinculadas a la inclusión digital en países de América Latina y en especial Cuba y análisar el comportamiento de este fenómeno en el área y las acciones realizadas por los diferentes gobiernos permitiendo mostrar cómo se ha comportado el desarrollo de políticas y proyectos de inclusión digital en América Latina y Cuba.

Método. Para profundizar en este análisis, se realizó una revisión bibliográfica que permitió ubicarnos en un contexto teórico-conceptual de este fenómeno. Se consultaron bases de datos internacionales que facilitaron el acceso a información que permitió arribar a varias conclusiones.

Conclusiones. A pesar de los esfuerzos realizados para disminuir la brecha digital, esta crece en la medida que se desarrollan nuevas tecnologías afectando a la población de menos recursos, se percibe también una tendencia en el crecimiento del uso de las TIC's en Cuba y América Latina.

\section{Palabras clave}

Inclusión digital ; Políticas públicas ; América Latina ; Cuba

\section{Public policies for digital inclusion: The case of Latin America and Cuba}

Abstract

Objective. This work deals about the development of public policies related to digital inclusion in Latin America, especially Cuba.

Method. A behavioral analysis of this phenomenon in the area and the actions taken by different governments allowed to show how the development of policies and digital inclusion projects in Latin America and Cuba have behaved. In order to deepen this analysis, a bibliographical review that put us on a theoretical and conceptual context of this phenomenon was performed. International databases that facilitated access to information that let us reach several conclusions were consulted.

Conclusions. Concluding that, despite the efforts made to decrease the digital breach, it increases as new technologies are developed affecting the population with fewer resources, is also reflected a trend in the growth of the use of TIC' $\mathrm{s}$ in Cuba and Latin America.

\section{Introducción}

El desarrollo alcanzado por la ciencia en el campo de la información y la comunicación devenida de los adelantos en las disciplinas de la informática, la electrónica, las telecomunicaciones, las tecnologías de satélites, la tecnología móvil, entre otros, representó un enorme avance en el desarrollo de la sociedad actual, la cual pasó de una sociedad meramente industrial, a una sociedad basada en el consumo elevado de la información, es por ello, que se le ha dado a llamar "Sociedad de la Información y el Conocimiento". 
Esta realidad influyó de tal manera en el quehacer de la sociedad que ha transformado la forma en que las personas viven, trabajan e interactúan. El surgimiento y desarrollo de la web, redes sociales, aplicativos móviles, etc. han llevado a planos socioculturales la utilización de las tecnologías de la información y comunicación (con internet como su más fiel exponente).

En la actualidad estas tecnologías de la información y las comunicaciones (TIC) devienen armas estratégicas para el desarrollo económico, político y social de cualquier nación. Este fenómeno ha llegado a tal punto, que podemos hablar de "[...] países info-ricos y países info-pobres [...]" (Sebastián, Méndez y Rodríguez, 2000, p.26), estimulado esto, por el poder adquirido por la información como valor económico ante la globalización mundial. Pero, si así de rápido se desarrollan estas las TICs, así de rápido crece la brecha digital entre comunidades, estados y países, entendida esta, como las desigualdades socioeconómicas en la accesibilidad a esas tecnologías.

La democratización del acceso a las tecnologías de la información y la comunicación, resulta la única vía posible para disminuir esta desigualdad, posibilitando así, que las personas consigan incorporar a sus rutinas diarias la utilización de estas tecnologías, de sus servicios, contenidos y aplicaciones, pero fundamentalmente que sean capaces de usarlas y darles un sentido útil en sus labores diarias. Este fenómeno que abarca aspectos del desarrollo de políticas sociales en relación al uso igualitario y equitativo de las nuevas tecnologías de la información y la comunicación por parte de la sociedad, se denomina Inclusión Digital y fue considerado por la UNESCO como un derecho humano.

Si bien no es suficiente colocar un computador en las manos de un campesino, de un obrero, de un adulto de la tercera edad; es preciso capacitar a esas personas para que puedan hacer un verdadero uso de estas tecnologías, es preciso proveer una infraestructura que garanta el acceso a las diferentes fuentes de información. Es por ello que resulta de gran importancia la creación de proyectos y políticas públicas, dirigidas a garantizar la inclusión digital de todos los miembros de la sociedad, sin distinción de razas, niveles de escolaridad, grupos etarios, ni ninguna otra forma de segregación.A bibliometria, conceituada por Sá (1978, p. 1)

De acuerdo con la UNESCO (2007), estas políticas se definen como "[...] políticas de estado que buscan disminuir la brecha de acceso, uso y aprovechamiento de la información, y que al mismo tiempo, establecen acciones para asegurar el acceso y preservación de la misma, ya sea pública o privada [...]"

Por otra parte Marquéz y Bueno (2005, p.) definen las políticas públicas como "[...] decisiones tomadas por el gobierno, que brindan soluciones a problemas identificados en determinados sectores de la población, de acuerdo con el análisis de factores económicos, políticos y simbólicos [...]"

Para los efectos de este trabajo se considerará como Políticas públicas aquellas acciones concretas de los gobiernos, dirigidas a enfrentar un problema social, o transformar cualquier aspecto de la realidad en un sentido determinado, privilegiando así aun grupo determinado de la sociedad.

Son las fuerzas políticas (partidos, grupos de presión, grupos parapartidistas, movimientos estudiantiles), sectores públicos, privados y cívicos los encargados de colocar la inclusión digital como "[...]punto fundamental del orden del día de la justicia social y los derechos humanos[...]"fomentando "[...]nuevos espacios para la tolerancia y la comprensión y contrarrestar los intentos de personas y grupos que buscan imponer valores, costumbres o creencias en el mundo digital y, que, en última instancia, afectan también a zonas no conectadas. [...]" López (2009, p 116)

La creación de una política pública de inclusión digital, como bien plantean Guerra y Jordán (2010, p.9) va estar condicionada por factores exógenos vinculados a:"[...]el nivel de desarrollo del país, su estabilidad y orientación política, así como el nivel de toma de conciencia sobre la importancia de la sociedad de la información[...]" y a factores endógenos "[...]sujetos a decisiones políticas y a las resoluciones del poder ejecutivo, se destacan el grado de participación y consenso que se pretenda lograr, el nivel jerárquico de las decisiones políticas y del organismo responsable, la calidad de la gestión administrativa y la disponibilidad de recursos[...]"

Ahora bien, el desarrollo de políticas públicas en el mundo contemporáneo se hace cada vez más necesario y complejo, con la confirmación de modelos políticos cada vez más neoliberales y orientados al mercado, los actores políticos encuentran cada vez más obstáculos en la creación de políticas públicas de inclusión social, que traten al ciudadano como un ente social y no como un mero cliente. 
En el caso de las políticas públicas de inclusión digital las cada vez más crecientes necesidades humanas, el desarrollo tan acelerado de las TIC, la falta de recursos y en ocasiones la poca voluntad de algunos gobiernos, dificulta que puedan ser diseñadas políticas públicas que repercutan de forma positiva en la utilización de estas tecnología en el mejoramiento de la calidad de vida del ciudadano común.

\section{Inclusión digital e políticas públicas en América Latina}

El masivo uso de las tecnologías de la información y la comunicación en los últimos tiempo, ha provocado un cambio de pensamiento y principalmente una importante transformación en la forma de actuar y relacionarse de las personas. Este fenómeno debe ser tomado en cuenta por los Estados, por dos razones fundamentales; una es, la obligación de los gobiernos con la reducción de la desigualdad existente en la sociedad, en cuanto al acceso de la misma a estas tecnologías, y por otro lado, la importancia del papel del sector público para la transformación de la sociedad hacia un nuevo modelo de organización social y productiva, donde se aprovechen las oportunidades que brindan el uso de las TIC.

Sin embargo, una de los principales cuestionamientos, va dirigido a, como están abordando estos temas y qué están haciendo los gobiernos para cumplir con estas obligaciones, si estamos, como bien plantea Elinett Wolf (2008, p20) “ [...]en una sociedad caracterizada, entre muchas variables, por la dominación, los individuos que someten o han sometido son los que tienen acceso a la información y al conocimiento y eso les ha dado ventajas comparativas frente a los otros[...]"

Según CECCHINI (2005,p.5)

[...]La llamada "brecha digital" interna de los países es determinada por características de los usuarios tales como el ingreso, nivel educativo y ubicación geográfica, e influenciada por otras características socio-económicas tales como el género, edad y etnia. En el ámbito de las actividades productivas, influye el tamaño de las empresas y su pertenencia al sector formal o informal. Aprovechar las oportunidades digitales depende por lo tanto no sólo de la presencia de una infraestructura de bajos costos, sino también de políticas públicas que tengan en cuenta los obstáculos enfrentados por los pobres [...].

Este es un tema que viene preocupando a todos los actores políticos de la región, ya desde el establecimiento de los Objetivos de Desarrollo del Milenio (ODM), y la realización de la Cumbre Mundial sobre la Sociedad de la Información (CMSI) en Ginebra (2003) y Túnez (2005). Es por ello que surge "El Plan de acción regional sobre la sociedad de la información en América Latina y el Caribe (eLAC)", documento que establecía, el compromiso de los gobiernos de trabajar para la eliminación de la brecha digital y por la creación de una sociedad de información más inclusiva. Existen varios momentos en el proceso de construcción de este plan:

"[...] el Plan de acción de la sociedad de la información en América Latina y el Caribe, conocido como eLAC2007. La segunda fase del Plan regional, eLAC2010, aprobada durante la Segunda Conferencia Ministerial sobre la Sociedad de la Información en América Latina y el Caribe realizada en San Salvador, El Salvador, en febrero de 2008. En el marco de la Tercera Conferencia Ministerial sobre la Sociedad de la Información en América Latina y el Caribe que tuvo lugar en Lima, Perú, en noviembre de 2010, se estableció la tercera fase del Plan regional, eLAC2015[...]. (CEPAL, 2013, p. 11)

Existen en América Latina varios proyectos y programas dirigidos a aprovechar las ventajas que ofrecen las (TIC) para la disminución de la desigualdad en el acceso a las TIC, intentando mejorar el acceso de sectores con bajos recursos a estas tecnologías y la capacitación para un mejor uso y aprovechamiento de sus ventajas. Según Amezquita (2009, p160) Internet y la telefonía se habían convertido en las dos principales TIC para países en desarrollo, a pesar de la brecha en acceso y cobertura. Ya desde el año 2011 existen países que se han tornado en líderes regionales en el uso de las TIC (Chile, Argentina, Brasil y México, seguidos por Colombia y Perú).

Entre los principales ámbitos de acción que están propuestos en el último Plan de Acción eLAC2015, se encuentran: a) mejorar el acceso e inclusión digital; b) trabajar para la creación de capacidades y de conocimientos y por consecuente lograr una mejora en el desarrollo de los procesos productivos y de innovación; c) aumentar la participación de gobiernos electrónicos para mejorar transparencia y eficiencia pública; d) crear instrumentos de política; e) gestión de un entorno habilitador; f) medio ambiente; g) seguridad social; h) una institucionalidad para una política de estado. 
En el cuadro I se muestran algunas de las iniciativas implementadas por los gobiernos para dar cumplimiento a las metas trazadas en cada uno de los ámbitos de acción, tómese en cuenta, que fue considerado como iniciativa un plan o proyecto TIC.

\begin{tabular}{|l|l|}
\hline \multicolumn{1}{|c|}{ País } & \multicolumn{1}{c|}{ Iniciativa } \\
\hline Argentina & $\begin{array}{l}\text { Plan Nacional Argentina Conectada } \\
\text { Centro Nacional de Datos y Red Federal de Fibra Óptica }\end{array}$ \\
\hline Barbados & Plan Estratégico de TIC de Barbados \\
\hline Brasil & $\begin{array}{l}\text { Plan Nacional de Banda Ancha } \\
\text { Proyecto Ciudades Digitales } \\
\text { Red Óptica del Gobierno Federal }\end{array}$ \\
\hline Chile & $\begin{array}{l}\text { Estrategia Digital 2007 - 2012 } \\
\text { Red de Conectividad y de Comunicaciones del Estado (INTRANET del Estado) }\end{array}$ \\
\hline Colombia & $\begin{array}{l}\text { Plan de Banda Ancha Vive Digital } \\
\text { Proyecto Nacional de Fibra Óptica } \\
\text { Red de Alta Velocidad del Estado Colombiano RAVEC }\end{array}$ \\
\hline Costa Rica & Plan Nacional de Desarrollo de las Telecomunicaciones \\
\hline Cuba & Programa Rector de la Informatización de la Sociedad Cubana \\
\hline Ecuador & Plan Nacional de Desarrollo de Banda Ancha \\
\hline Guatemala & Agenda Nacional de la Sociedad de la Información y el Conocimiento \\
\hline Jamaica & e-Powering Jamaica \\
\hline México & $\begin{array}{l}\text { Red Dorsal Nacional de Fibra Óptica, Red NIBA } \\
\text { Agenda Digital.mx }\end{array}$ \\
\hline Panamá & Agenda Digital Estratégica de la Autoridad para la Innovación Gubernamental \\
\hline Paraguay & Plan Director TIC 2012 \\
\hline Perú & $\begin{array}{l}\text { Plan de Desarrollo Digital de la SI en el Perú, Agenda Digital 2.0 } \\
\text { Plan Nacional para el Desarrollo de la Banda Ancha en el Perú }\end{array}$ \\
\hline $\begin{array}{l}\text { República Bolivariana } \\
\text { de Venezuela }\end{array}$ & $\begin{array}{l}\text { Plan Nacional de Telecomunicaciones, Informática y Servicios Postales 2007- } \\
\text { 2013 }\end{array}$ \\
\hline $\begin{array}{l}\text { República } \\
\text { Dominicana }\end{array}$ & Red de Fibra Óptica Estrategia Nacional de Desarrollo 2030 \\
\hline Uruguay & $\begin{array}{l}\text { Plan Ceibal Centros MEC } \\
\text { Programa Salud.uy } \\
\text { REDuy }\end{array}$ \\
\hline
\end{tabular}

Cuadro 1 : Iniciativas Gubernamentales para el cumplimiento del Plan de Acción eLAC2015 Fuente: Monitoreo del Plan de Acción eLAC2015

A continuación se presentan algunos datos, que muestran los resultados obtenidos por los países de la región en el cumplimiento de estas metas.

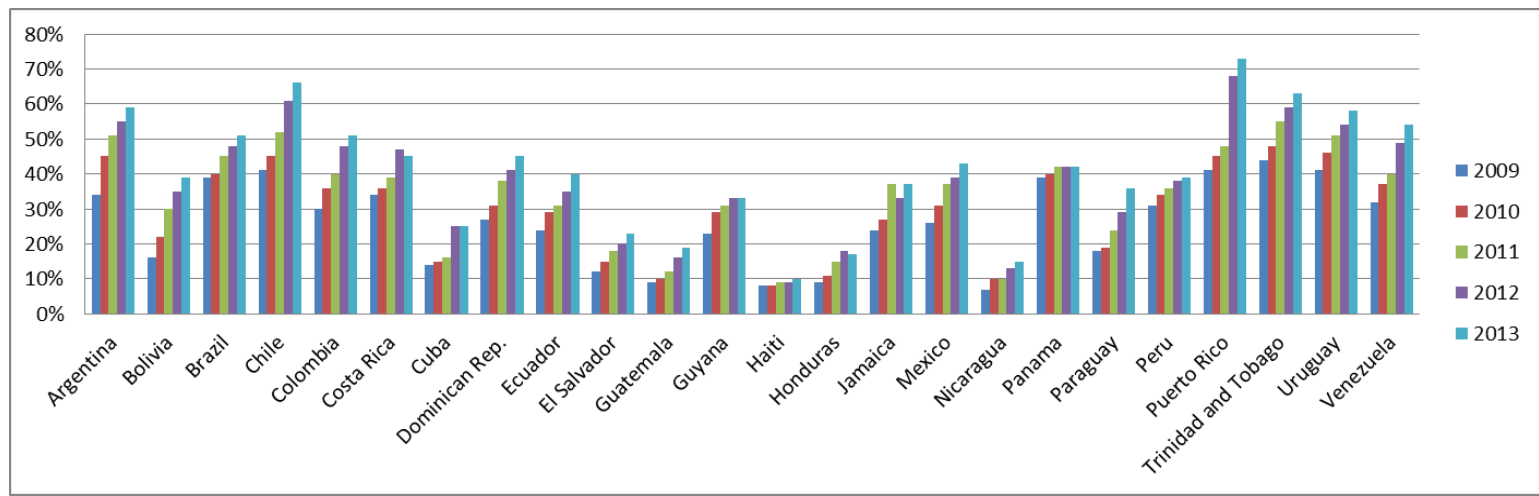

Figura 1 : Porcentaje de usuarios individuales con acceso a internet en el período 2009-2013 Fuente: Elaboración propia con datos de la World Telecommunication/ICT Indicators DatabaseInternational Telecommunications Union(ITU) 
En la Figura 1 se muestra el porciento de crecimiento por países de usuarios individuales con acceso a internet. En la misma se refleja un crecimiento en todos los países del acceso individual a internet, sin embargo, al mismo tiempo reflejan que en el $66,6 \%$ de los países, menos del $50 \%$ de la población tiene acceso a este servicio.

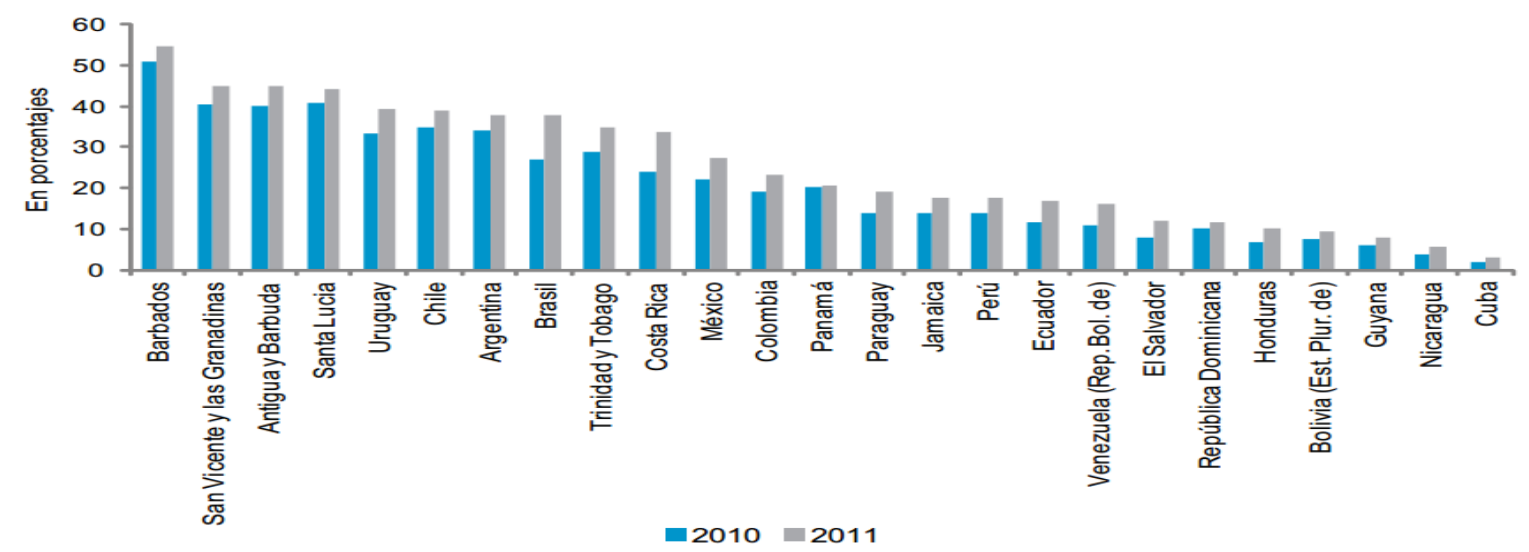

Figura 2 : Hogares con acceso a internet en el propio hogar, 2010-2011 Fuente: Monitoreo del Plan de Acción eLAC2015

En la Figura 2 se demuestra un progresivo aumento en la penetración del servicio de internet en los hogares de todos los países, aunque en países como Panamá y Cuba este aumento no fue tan significativo, siguiendo la misma tendencia del gráfico anterior al mostrar que excepto en Barbados, hasta el 2011 ningún país había conseguido sobrepasar el $50 \%$ de familias con acceso a internet en su propio hogar.

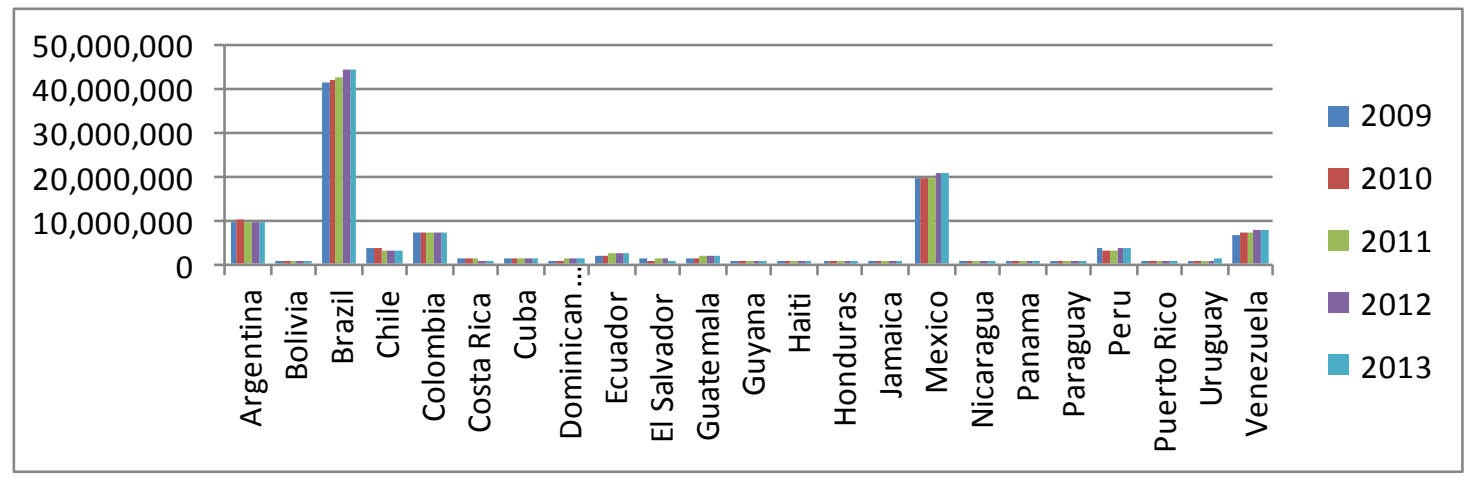

Figura 3 Cantidad de usuarios subscritos al servicio de telefonía fija en el período 2009-2013

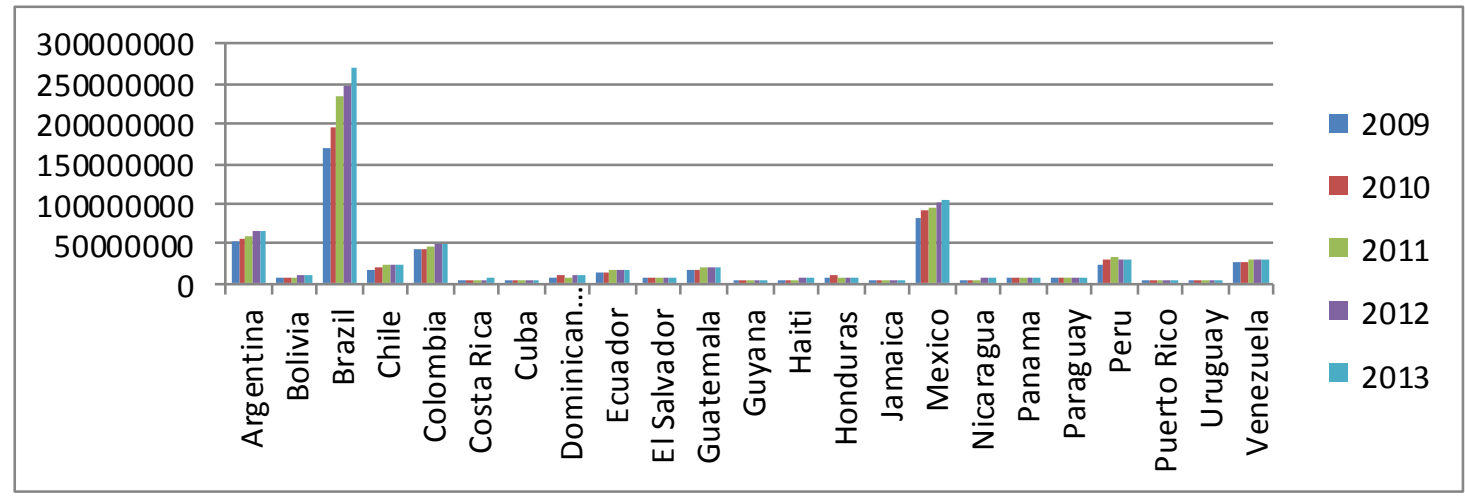

Figura 4 Cantidad de usuarios subscritos al servicio de telefonía móvil en el período 2009-2013 Fuente: Elaboración propia 
En las Figuras 3 y 4 se presentan el acceso de los usuarios a los servicios de telefonía fija y móvil, destacándose un crecimiento superior de la móvil con respecto a la fija, tendencia esta que se refleja en todo el mundo.

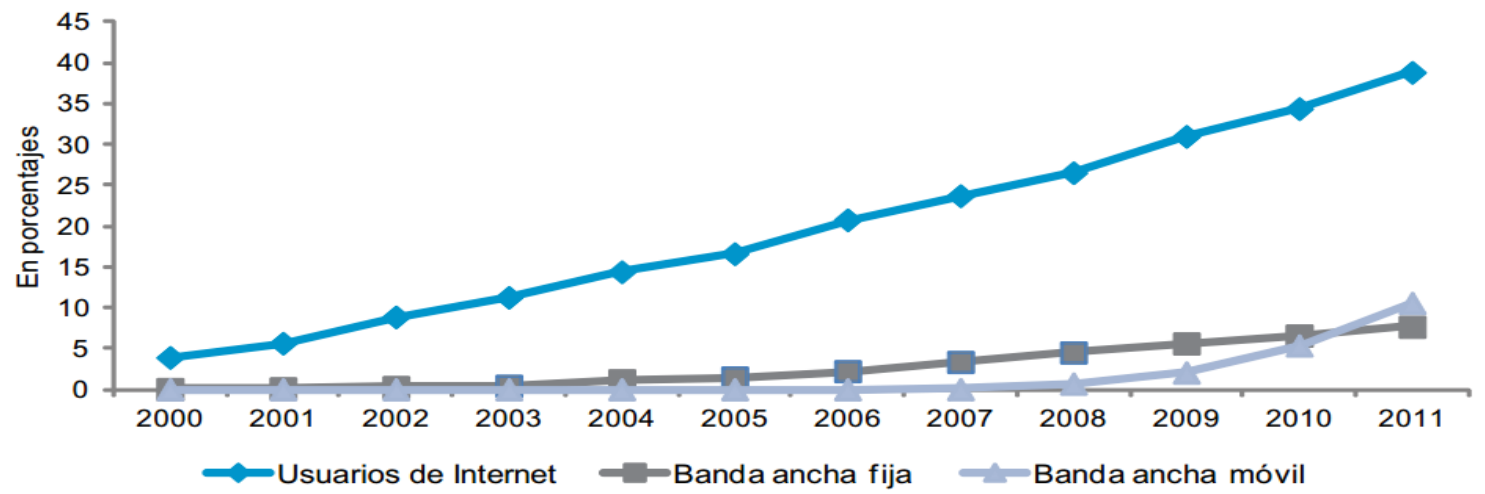

Figura 5: Evolución de la penetración de usuarios de internet, banda ancha fija y banda ancha móvil (por cada 100 habitantes), 2000-2011

Fuente:Monitoreo del Plan de Acción eLAC2015

En la Figura 5 se demuestra, como en el período contemplado entre 2000 y 2011 los niveles de acceso a internet fueron creciendo gradualmente, en el caso de la telefonía móvil a partir del año 2007, aun así, este aumento no elimina la brecha digital y la desigualdad en el acceso a estas tecnologías, ya que, por cada 100 habitantes solo un promedio de 39 tenía acceso a internet, 8 a para banda ancha fija y 11 para banda ancha móvil.

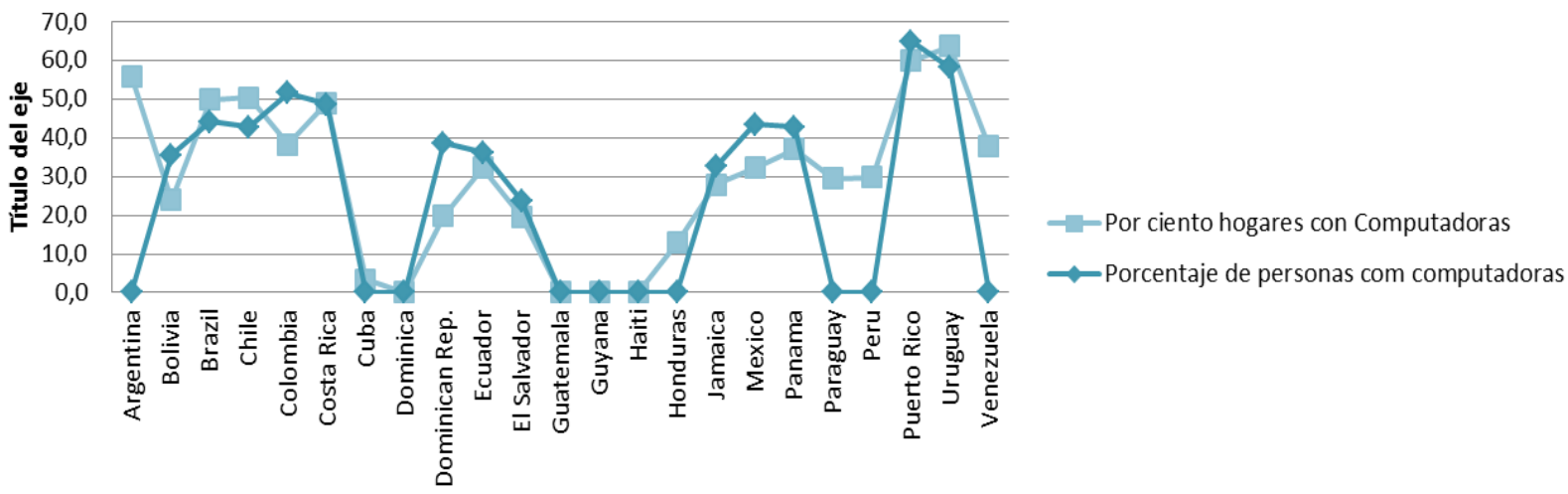

Figura 6 : Por cientos hogares y usuarios con computadoras.

Fuente: Elaboración propia con datos de la World Telecommunication/ICT Indicators DatabaseInternational Telecommunications Union(ITU)

En la Figura 6 se puede observar, que en muchos países de la región aun los niveles de acceso a este tipo de tecnología alcanzan a menos del $50 \%$ de la populación, lo que indica que a pesar de los esfuerzos realizados por algunos gobiernos, estos resultan insuficientes todavía.

Si reflexionamos al respecto de todos estos datos podemos percibir que, los gobiernos de la región deben trabajar cada vez más en: a) estructurar y fomentar cada vez más, planes que garanticen el acceso y que permitan disminuir la todavía existente brecha digital; b) aumentar el intercambio de experiencias con países de la región con buenos resultados en estos procesos; c) colocar metas con valores similares a la media de la región y velar por que sean cumplidas cada una de ellas. 


\section{Políticas públicas e Inclusión digital en Cuba}

Cuba es un país ubicado en América Central, en la zona del caribe, es una isla con una población de alrededor de 11.167.325 personas según la Oficina Nacional de Estadísticas e Información (ONEI, 2012), con un gobierno de carácter socialista y miembro de organizaciones internacionales tales como: CELAC, ALADI, ALBA, G-77, NOAL, ONU, OEI, AEC, Grupo de Río.

El gobierno cubano, desde el triunfo de la revolución en el año 1959 dedicó grandes esfuerzos a la búsqueda de recursos, alternativas y soluciones para los problemas económicos y sociales, en pos de garantizar los servicios básicos y potenciar los distintos sectores de la sociedad a través de una política social justa, participativa y de igualdad de posibilidades, oportunidades y acceso.

El camino iniciado por Cuba desde hace más de cinco décadas, ha tenido tropiezos, insuficiencias y errores, pero el Estado ha mantenido como principal objetivo la atención a los sectores poblacionales más vulnerables, privilegiando los intereses colectivos sobre los individuales. El desarrollo de Políticas públicas dirigidas al beneficio de la sociedad, ha sido una constante de gobierno cubano durante todo ese período, buscando garantizar una distribución equitativa e igualitaria de sus recursos que llegue a todos sus ciudadanos por igual.

Una revolución tecnológica, centrada en torno a las tecnologías de la información, está modificando la base material de la sociedad a un ritmo acelerado y al igual que en América Latina y el resto del mundo, Cuba vive un momento expectante, y está ante la oportunidad de dar un salto tecnológico que permita crecer económicamente y mejorar la calidad vida de la población y las telecomunicaciones juegan un papel fundamental en este proceso, pues favorecen una mayor inclusión social y fortalecen el desarrollo cultural del pueblo.

Pero para hablar de este fenómeno, se hace necesario abordar 2 elementos esenciales que condicionan y están presentes en todas las acciones que se vienen desarrollando para dar el gran salto. La primera está relacionada a la situación económica y política del país en los últimos años; y la segunda está vinculada a la política de embargo económico que los Estados Unidos tienen implantada contra Cuba.

Si bien, hasta mediados y finales de la década de los 80, Cuba mantenía un ritmo de desarrollo considerado como muy bueno, basado esto fundamentalmente en su favorable situación como de miembro del Consejo de Ayuda Mutua Económica (CAME), -organización integrada por los países del extinto bloque socialista liderado por la Unión Soviética-, y del cual dependía el 85\% de la economía cubana, ya para comienzo de la década de los años 90 esta situación cambió abruptamente. Con la caída del campo socialista, Cuba vio desaparecer su mayor fuente de ingreso y tuvo que abrir a nuevas variantes económicas que le permitieran mantener el modelo hasta esa época construido. Es así que comienza el llamado período especial, etapa de la década de los 90 donde el gobierno cubano atravesó la mayor crisis económica de su historia y que decidió enfrentar sin perder su carácter socialista y sin abandonar las conquistas sociales alcanzadas hasta ese momento.

- Acceso a una alimentación básica;

- Asegurar el derecho ciudadano a la salud y la educación; ingreso adecuado para quienes se jubilan o requieren de un apoyo económico de la sociedad;

- Fuentes de empleo; protección y descanso del trabajador;

- Una vivienda confortable, preferiblemente de propiedad propia;

- Lograr una sociedad progresivamente más justa y solidaria. (ECURED, 2012)

El otro lado de la moneda se refiere al Embargo Económico y financiero que los Estados Unidos tienen implantado contra Cuba (también llamado como bloqueo económico) impuesto desde octubre de 1960 hasta la actualidad y que lo convierte en el más largo embargo económico implementado contra país algún. Este embargo adquirió el carácter de ley con el propósito de mantener las sanciones contra la República de Cuba.

Estas medidas prohíben a las empresas, ciudadanos y a las filiales extranjeras de compañías estadounidenses de comercializar con Cuba, además de imponer grandes multas a aquellas empresas extrajeras que para comercializar con Cuba utilicen el Dollar Americano como moneda de cambio. 
Es en esta situación que se desarrollan en Cuba proyectos de desarrollo que le permitan al país alcanzar un desarrollo, pudiera decirse considerable en la eliminación de la brecha digital, sobre todo, cuándo para ello depende fórmulas, que permitan evadir el embargo económico y que resultan siempre más caras. Para tener una idea del impacto que puede llegar a tener este embargo sobre el desarrollo tecnológico de Cuba, en estos momentos un país con más de once millones de habitantes, tiene autorizados apenas, para acceder Internet $65 \mathrm{Mbps}$ de ancho de banda para la salida y $124 \mathrm{Mbps}$ para la entrada vía satélite. Lo más absurdo es que, con los adelantos y los niveles de penetración de internet en la vida de los ciudadanos en todo el mundo, un solo usuario corporativo o incluso particular, en Europa, Asia o Estados Unidos, tiene hoy una velocidad de conexión a la red de redes mayor que la que tiene Cuba.

Cuba por causa del embargo, está prohibida de conectarse a los cables de fibra óptica que pasan cerca de sus fronteras marítimas, como lo demuestra la Figura 7. Siendo solo autorizado en 1996, su conexión a través de satélite (conexión mucho más cara que por fibra óptica) tras mucha presión del gobierno cubano ante las organizaciones mundiales.

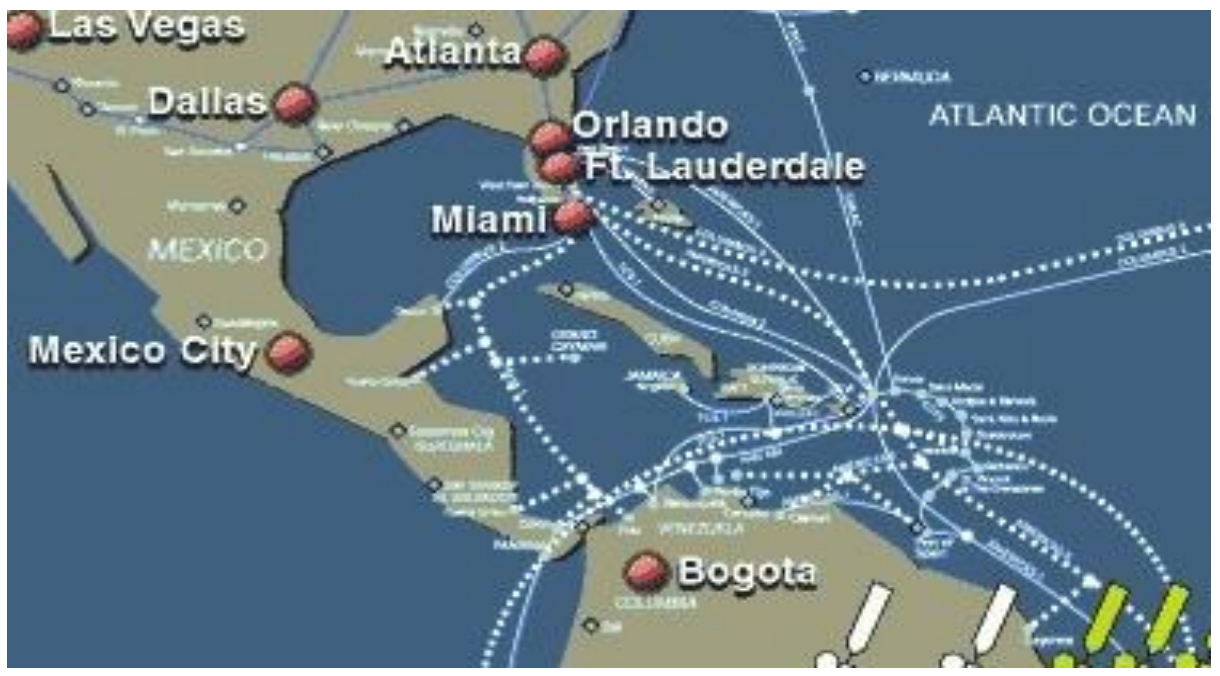

Figura 7 Mapa de fibra óptica de América Central y el Caribe

Fuente: La Joven Cuba: Un Blog de Jóvenes Universitarios que opinan sobre la Realidad Cubana.

Sin embargo, esta realidad no impidió que el gobierno adoptara medidas para garantizar el acceso de la población a las nuevas TIC's. En la tabla se muestran algunas de las acciones realizadas por el gobierno cubano con el objetivo de acercar al pueblo, no solo los avances de las nuevas TIC's, así como capacitarlo para hacer un mejor uso de estas nuevas herramientas.

Tabla 1: Acciones realizadas por el gobierno cubano para garantizar la inclusión digital de la populación (Continua).

\begin{tabular}{|l|c|}
\hline \multicolumn{1}{|c|}{ Epoca } & Acciones \\
\hline $\begin{array}{l}\text { Comienzo de la } \\
\text { Década de los } 80\end{array}$ & $\begin{array}{c}\text { El gobierno cubano comienza a introducir en las escuelas el uso de las computadoras e } \\
\text { incluye en los programas de carrera con carácter obligatorio, la presencia de asignaturas } \\
\text { que enseñen el uso de las nuevas tecnologías. }\end{array}$ \\
\hline Año 1987 & $\begin{array}{c}\text { Laa Unión de Jóvenes Comunistas (UJC) desarrolla un proyecto para la creación de una } \\
\text { red de telecentros, llamados de Joven Club de Computación, con el objetivo de alfabetizar } \\
\text { a la sociedad en el uso de las TIC's (603 telecentros) con una capacidad promedio de 18 } \\
642 \text { usuarios e un total de 9 331 PC's }\end{array}$ \\
\hline $\begin{array}{l}\text { Desde } 1987 \text { hasta } \\
\text { el año } 2011\end{array}$ & $\begin{array}{r}\text { Fueron impartidos más de 60 000 cursos de forma gratuita, para capacitar a la población } \\
\text { en el uso de las TIC's, con un total de 3 043 299graduados. }\end{array}$ \\
\hline
\end{tabular}

Fuente: Elaboración Propia. 
Tabla 2: Acciones realizadas por el gobierno cubano para garantizar la inclusión digital de la populación (Conclusão).

\begin{tabular}{|c|l|}
\hline Epoca & \multicolumn{1}{c|}{ Acciones } \\
\hline \multirow{3}{*}{ Entre 1997 y 2003} & $\begin{array}{l}\text { Fueron propuestas } 2 \text { políticas públicas relacionadas al uso de las TIC's; } \\
\text { - Política Nacional de Información (PNI) } \\
\text { - Política de Informatización da Sociedad, siendo únicamente esta segunda reconocida } \\
\text { oficialmente por el gobierno }\end{array}$ \\
\hline Año 2000 & $\begin{array}{l}\text { Se crea el Ministerio de Informática e Comunicaciones (MIC) cuya función principal es la } \\
\text { de fomentar el uso de las Tecnologías de la Información y las Comunicaciones (TIC) en } \\
\text { función del desarrollo de la economía nacional, la sociedad y al servicio del ciudadano. } \\
\text { Además tiene la responsabilidad de garantizar la fiabilidad, estabilidad, seguridad, } \\
\text { invulnerabilidad y soberanía de dichas tecnologías. }\end{array}$ \\
\hline \multirow{2}{*}{2001} & $\begin{array}{l}\text { Es aprobado el proyecto de informatización de la educación, cuyo objetivo principal es el } \\
\text { de informatizar todos los procesos educativos en todos los niveles de enseñanza. }\end{array}$ \\
\hline \multirow{2}{*}{2007} & $\begin{array}{l}\text { Se crea el "Proyecto Futuro", con dos objetivos: } \\
\text { - Informatizar el país; } \\
\text { - Desarrollar la industria nacional de Software. } \\
\text { Para alcanzar esos objetivos fue creada en el mismo año la Universidad de Ciencias } \\
\text { Informáticas UCl. }\end{array}$ \\
\hline \multirow{2}{*}{2014} & $\begin{array}{l}\text { Se firmó en Caracas un acuerdo para la creación de una empresa mixta, conformada por } \\
\text { la entidad estatal de telecomunicaciones de Venezuela (TELECOM) y la cubana } \\
\text { TRANSBIT. Con el objetivo de la puesta en funcionamiento de un cable submarino de } \\
\text { fibra óptica que conecta a ambas naciones, así como a otros países del área, y que } \\
\text { permitirá una mayor independencia en las comunicaciones. }\end{array}$ \\
\hline $\begin{array}{l}\text { Se abren salas de navegación con acceso a internet para toda la población y se } \\
\text { disponibiliza el acceso a internet desde los móviles. }\end{array}$ \\
\hline
\end{tabular}

Fuente: Elaboración Propia.

A pesar de los problemas económicos que enfrenta, el gobierno cubano ha destinado grandes cantidades de recursos para el desarrollo tecnológico del país, para formar y capacitar a la población en el uso y manejo de las TIC's. a continuación se muestran algunos datos de cómo se ha reflejado estos esfuerzos en el desarrollo tecnológico del país.

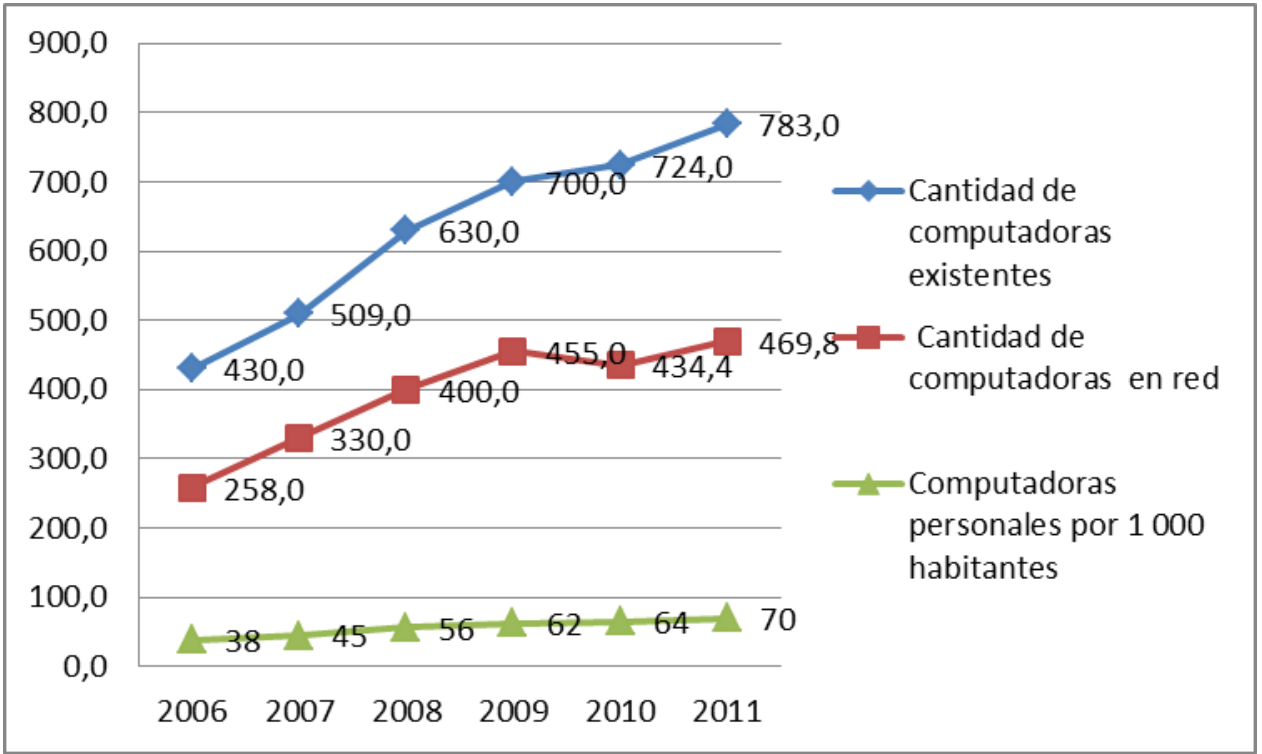

Gráfico 1 : Evolución de la cantidad de computadoras existentes y las conectadas en Red. Fuente: Elaboración propia con uso de los datos de la Oficina Nacional de Estadísticas e Información (ONEI). La unidad de medidas es en base a 1000. 


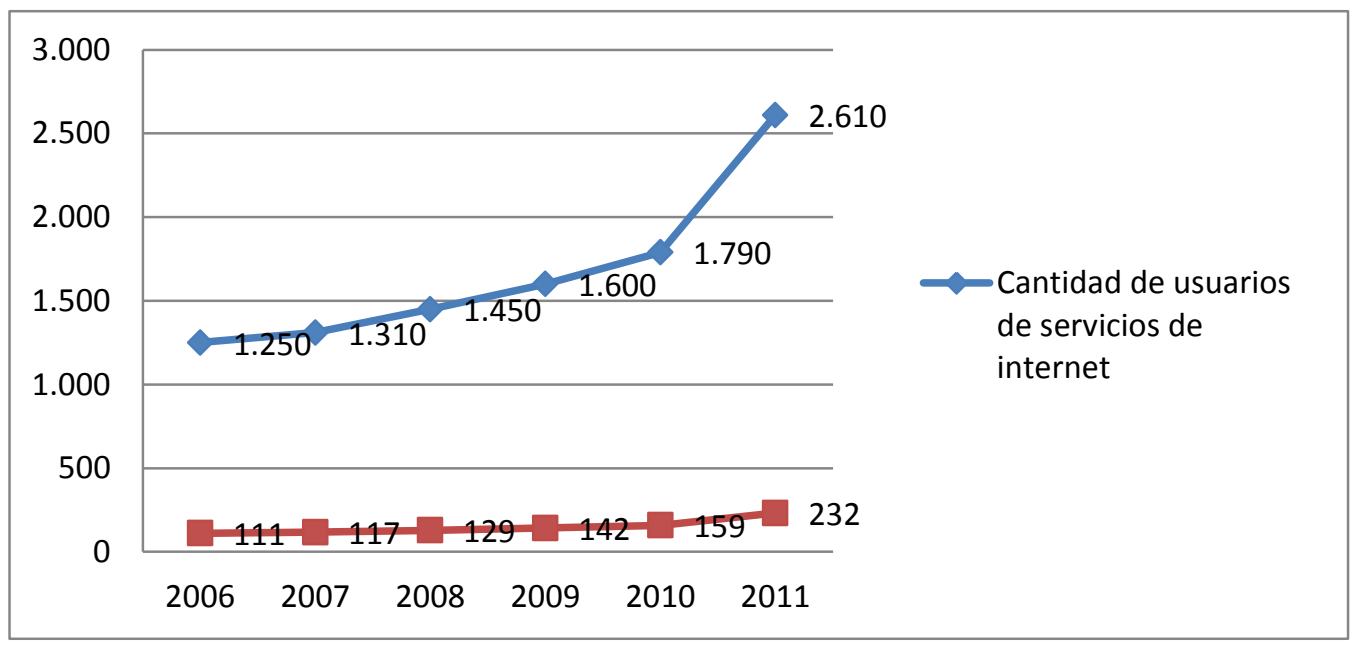

Gráfico 2 : Cantidad de usuarios con Acceso a internet.

Fuente: Elaboración propia con uso de los datos de la Oficina Nacional de Estadísticas e Información (ONEI)

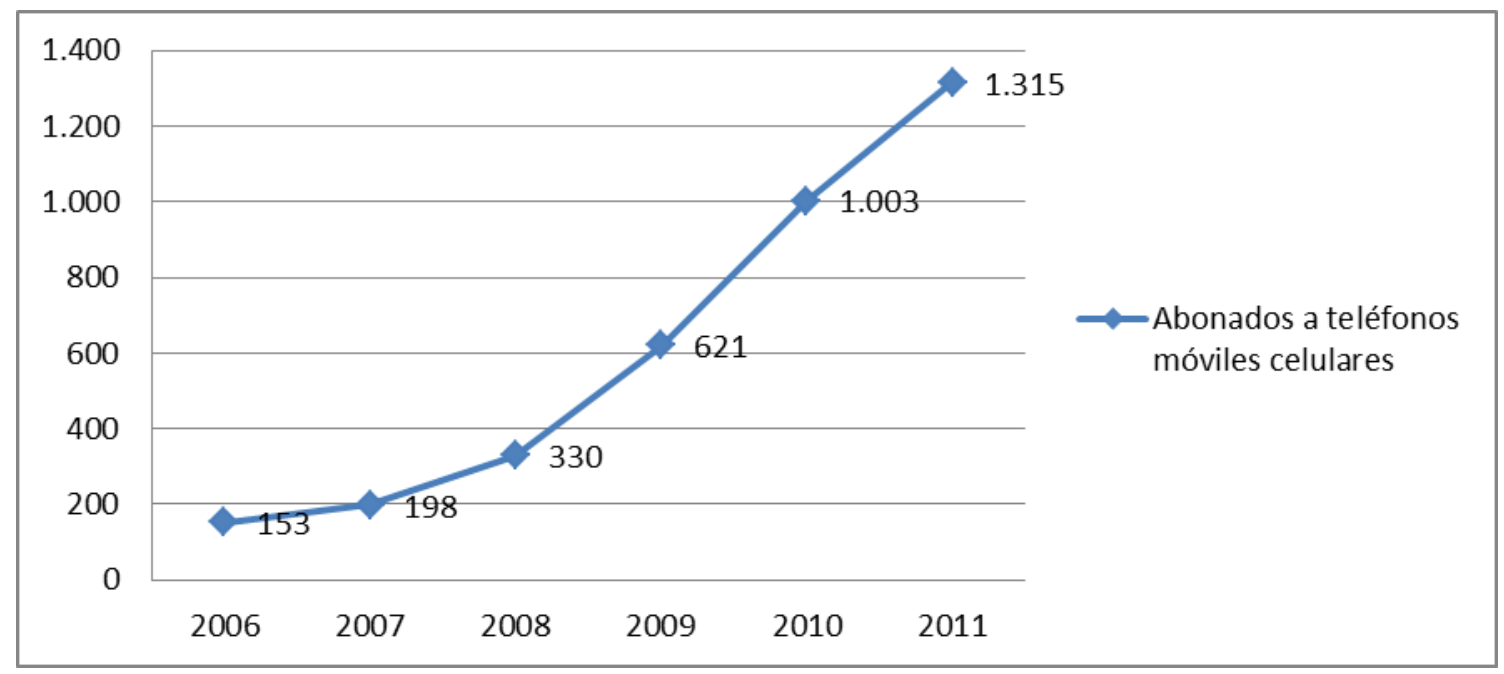

Gráfico 3 : Usuarios abonados a los servicios de telefonía móvil.

Fuente: Elaboración propia con uso de los datos de la Oficina Nacional de Estadísticas e Información (ONEI)

Según los datos mostrados en los Gráficos 1, 2 Y 3; Cuba es uno de los países con un per cápita de usuarios con acceso a internet, cantidad de computadoras por habitantes e índices de abonados a los servicios de telefonía móvil más bajos de todos los países pertenecientes a la CELAC, pero al mismo tiempo uno de los que más ha trabajado en desarrollar proyectos y acciones de inclusión digital.

\section{Consideraciones finales}

La lucha por eliminar las desigualdades sociales existentes en la humanidad, también se ve reflejada en el desarrollo de las tecnologías de la información y la comunicación, los niveles de exclusión digital de varios sectores sociales que están quedando fuera de los últimos adelantos en esta área, demuestra que a pesar de la tendencia de alza de los últimos años en los índices de penetración de estas tecnologías en la sociedad y los esfuerzos realizados por disminuir la brecha digital, en la medida que se desarrollan las nuevas tecnologías esta brecha crece. 
En la Región de América Latina los datos demuestran que el acceso a las TIC's lleva una tendencia creciente, pero que aún resulta insuficiente, tenemos en cuenta que más del 50\% la populación en la mayoría de los países aun no tienen acceso estas tecnologías. Cuba dentro de esta región es uno de los países con más bajo índice de penetración hacia el interior de su población, fenómeno que tiene sus causas en los bajos niveles de desarrollo económico del país, lo cual incide en la posibilidad del Estado en invertir en el acceso, mantenimiento y actualización de una tecnología que todos los días está mudando, sin embargo, a pesar de las dificultades el gobierno cubano ha desarrollado varios proyectos de inclusión digital para poner estas tecnologías al alcance de toda la población.

\section{Referências}

AMÉZQUITA, C. Panorama de la Sociedad de la Información en América Latina (2000-2007). Revista Facultad de Ciencias Económicas: Investigación y Reflexión. V.17, n.2, p.151-170. 2009

CECCHINI, S. Oportunidades digitales, equidad y pobreza en América Latina: ¿Qué podemos aprender de la evidencia empírica? Publicación de las Naciones Unidas CEPAL - SERIE Estudios estadísticos y prospectivos. 2005, disponible en: http:// daccess-ddsny.un.org/doc/UNDOC/LTD/S05/010/81/PDF/S0501081. pdf?OpenElement, Consultado en: 25 de Agosto de 2014

CEPAL. Monitoreo del Plan de Acción eLAC2015. Cuarta Conferencia Ministerial sobre la Sociedad de la Información en América Latina y el Caribe, Montevideo, 3 a 5 de abril de 2013. Disponible en:

http://archivo.cepal.org/pdfs/2013/S2013111.pdf. Consultado en: 25 de Agosto de 2014

ECURED. Enciclopedia Cubana. Disponible en: http://www.ecured.cu/index.php/Cuba\#Econom.C3.ADa, Consultado en: 01 de septiembre de 2014

GUERRA, M. Y JORDÁN, V. Políticas públicas de Sociedad de la Información en América Latina: ¿una misma visión?. Comisión Económica para América Latina y el Caribe, Naciones Unidas. 2010

LÓPEZ, P. Inclusión Digital: Un Nuevo Derecho Humano. Educación y Biblioteca, julio/agosto № 172, p. 114-118. 2009

MÁRQUEZ M. T.; BUENO, C. Políticas públicas en Tecnologías de la Información. La penitencia antropológica. Revista Cuicuilco, v.12, n.35, p.37-57. 2005

ORGANIZACIÓN DE LAS NACIONES UNIDAS PARA LA EDUCACIÓN, LA CIENCIA Y LA CULTURA. Hacia la Construcción de Políticas Nacionales de Información: la Experiencia de América Latina. UNESCO. 2007

SEBASTIÁN, M. C., MÉNDEZ, E. Y RODRÍGUEZ, DAVID La necesidad de políticas de información ante la nueva sociedad globalizada. El caso español. Ciencia de Información, maio/ago, v. 29, n. 2, p. 22-36. 2000

WOLF, E. Inclusión Digital y Telecentros en América Latina. Fundación Telefónica, 2008. 


\section{Dados dos autores}

\section{Edgar Bisset Alvarez}

Doutorando no Programa de Pós-graduação em Ciência da Informação na Universidade Estadual PaulistaFaculdade de Filosofia e Ciência. Possui graduação em Informação Cientifico-Técnica y Bibliotecologia Universidade da Havana (2007) e mestrado em Bibliotecologia e Ciências de la Informação - Universidade da Havana (2012). Pertence à Associação Cubana de Biblioteconomia (ASCUBI), ao grupo de Trabalho GT-8 "Informação e Tecnologia" da Associação Nacional de Pesquisa e Pós-Graduação em Ciência da Informação (ANCB).

\section{ebicet@gmail.com}

\section{Angela Maria Grossi de Carvalho}

Professor Assistente Doutor da Universidade Estadual Paulista Júlio de Mesquita Filho (UNESP). Possui graduação em Comunicação Social - habilitação em Jornalismo pela Universidade de Sorocaba (1999), mestrado em Educação pela Universidade Metodista de Piracicaba (2005), doutorado em Ciência da Informação pela Universidade Estadual Paulista Júlio de Mesquita Filho (2010) e pós-doutorado em Comunicação pela Universidade de Sevilha-Espanha (2011). Atualmente é docente do curso de Jornalismo e do Programa de PósGraduação em Ciência da Informação (Unidade de Marília) da Universidade Estadual Paulista Júlio de Mesquita Filho (UNESP), pesquisadora do Laboratório de Estudos de Comunicação, Tecnologia e Educação Cidadã (LECOTEC-UNESP), coordenadora do projeto de extensão Portal Toque da Ciência. Membro do Conselho do Curso de Jornalismo.

angela@carvalho.jor.br

\section{Silvana Aparecida Borsetti Gregorio Vidotti}

Professora Assistente-Doutora em Regime de Dedicação Integral à Docência e à Pesquisa da Universidade Estadual Paulista Júlio de Mesquita Filho. Licenciada em Matemática pelo Instituto de Biociências, Letras e Ciências Exatas da UNESP (1986). Especialista em Ciência da Computação pelo Instituto de Ciências Matemáticas de São Carlos da USP (1987). Mestre em Ciências área de concentração - Ciências da Computação e Matemática Computacional - pelo Instituto de Ciências Matemáticas de São Carlos da USP (1993). Doutora em Educação - área de concentração Educação Brasileira - pela Faculdade de Filosofia e Ciências da UNESP (2001). Docente dos cursos de graduação em Arquivologia e Biblioteconomia e dos cursos de mestrado acadêmico e doutorado em Ciência da Informação da Unesp. vidotti@marilia.unesp.br

Recibido - Received : 2014-12-15

Aceptado - Accepted: 2015-03-30

\section{$(\mathrm{cc}) \mathrm{BY}$}

This work is licensed under a Creative Commons Attribution 4.0

United States License.

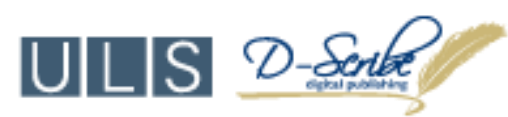

This journal is published by the University Library System of the University of Pittsburgh as part of its D-Scribe Digital Publishing Program and is cosponsored by the University of Pittsburgh Press. 